\title{
Autonomic arousal in a foreign language in the context of decision making
}

\author{
Mai Al-Khatib \& Charles R. Fletcher*
}

\begin{abstract}
We test emotional distancing in a second language (L2) by replicating an experiment by Keysar, Hayakawa, and An (2012) on making decisions under the framing effect (Kahneman and Tversky, 1979). With their participants' average Age of Acquisition (AoA) being around and beyond puberty, autonomic arousal was evident in native language (L1) but absent in L2. Our study showed no difference between L1 and L2 when AoA was around 4. However, when average AoA was around 7.7, autonomic arousal was evident in L1 but absent in L2, predicting an AoA threshold affecting L2 affective processing significantly earlier than puberty.
\end{abstract}

Keywords. bilingualism; language and affect; decision making; age of acquisition; Prospect Theory; the framing effect

1. Introduction. According to Prospect Theory, humans are irrational decision makers because they respond differently to equivalent situations when they are framed differently. When a dilemma is framed in terms of losses, people tend to choose the risky choice. But if it is framed in terms of gains, they show risk aversion (Kahneman \& Tversky,1979). When people make moral judgments, they are driven by a complex interaction of at least two forces: intuitive "automatic'" processes prompted by the emotional content of a given dilemma; and rational, effortful, controlled processes driven by the conscious evaluation of the potential outcomes (Costa et al.,2014; Green \& Haidt, 2002; Haidt, 2007; Baron, 1993). In other words, people have two modes of processing when making decisions: affective and cognitive, respectively.

Bilinguals were shown to make rational choices in L2 when faced by a moral dilemma (e.g. the trolley dilemma) (Costa et al.,2014). Keysar, Hayakawa, \& An (henceforth referred to as KHA) tested Prospect Theory on late bilinguals to establish that L2 reduces decision biases (2012). Three bilingual populations: English/ Japanese, Korean/ English, and English/ French L1/ L2 speakers were tested. The Average Age of Acquisition (AoA) in the three samples was between 12 and 17 years old with L2 learned in the classroom. The participants were asked to respond to a dilemma that is a modified version of the Asian Disease Problem. The original version states that a disease outbreak threatens a population of people and would be addressed by two programs with different certain consequences. The consequences are laid out in terms of either the gains of adopting one program over the other or the losses thereof (Tversky \& Kahneman, 1981); that is, the formulation is in terms of either how many people would be saved or how many would die. KHA modified the problem to look as shown in Table 1. Across the three samples, participants exhibited decision biases in L1 as predicted by Prospect Theory, but the bias was not evident in L2. This implied that late bilinguals have overridden decision bias due to framing because making decisions in a foreign language reduces emotional reaction (what we refer to as autonomous arousal) to the moral dilemma they were faced with. We believe that this implies more rationality of decision making in L2.

\footnotetext{
* We thank the German Jordanian University and Philadelphia University in Jordan for allowing us to recruit students for our experiments on their campuses. We also thank Ms. Eman AlNagi from Philadelphia University for facilitating data collection in experiment 2. From the Institute of Linguistics in the University of Minnesota, we thank Prof. Jason Overfelt and Prof. Hooi Ling Soh for their valuable feedback and insights. Mai Al-Khatib, University of Minnesota (alkh0020@umn.edu) \&Charles R. Fletcher, University of Minnesota (fletc002@umn.edu).
} 
We asked the following question: Would the KHA results obtain for a new bilingual population? That is, if we present a new sample of late bilinguals with the dilemma in Table 1, will they exhibit decision biases in L1 but not in L2? If so, then what is the AoA threshold for this to be observed? We replicated KHA's experiment on Arabic/ English bilingual university students in Jordan.

2. Method. We conducted KHA's experiment on two samples of L1/ L2 Arabic/ English college students in Jordan who differed significantly in AoA. In both samples, participants learned English mainly in classrooms and did not have a parent who was a native speaker of English. They were randomly assigned to four groups resulting from combining the language (English or Arabic) and frame (gain or loss) criteria. They responded to an online survey that we built using Qualtrics software, Version 05-2018 of Qualtrics. Copyright (C) 2019 Qualtrics. Qualtrics and all other Qualtrics product or service names are registered trademarks or trademarks of Qualtrics, Provo, UT, USA. https://www.qualtrics.com.

The participants were presented with the Asian Disease dilemma depicted in either Tables 1 or 2, depending on their assignment: Arabic - Gain Frame, Arabic - Loss Frame, English - Gain Frame, or English - Loss Frame. The survey collected demographic information such as age, gender, and the age of acquisition (AoA) of English. They were also asked to rate their own proficiency on a scale from 0 to 10 on both Arabic and English. Surveys in the Arabic condition were wholly presented in Arabic and in the English condition were wholly presented in English.

\section{English Gain Frame Version}

Recently, a dangerous new disease has been going around. Without medicine, 600,000 people will die from it. In order to save these people, two types of medicine are being made. If you choose Medicine A, 200,000 people will be saved.

If you choose Medicine $\mathrm{B}$, there is a $33.3 \%$ chance that 600,000 people will be saved and a $66.6 \%$ chance that no one will be saved.

Which medicine do you choose?

\section{English Loss Frame Version}

Recently, a dangerous new disease has been going around. Without medicine, 600,000 people will die from it. In order to save these people, two types of medicine are being made. If you choose Medicine A, 400,000 people will die.

If you choose Medicine B, there is a 33.3\% chance that no one will die and a $66.6 \%$ chance that 600,000 people will die.

Which medicine do you choose?

Table 1. A modified version of the Asian Disease problem (Keysar, Hayakawa, \& An, 2012)

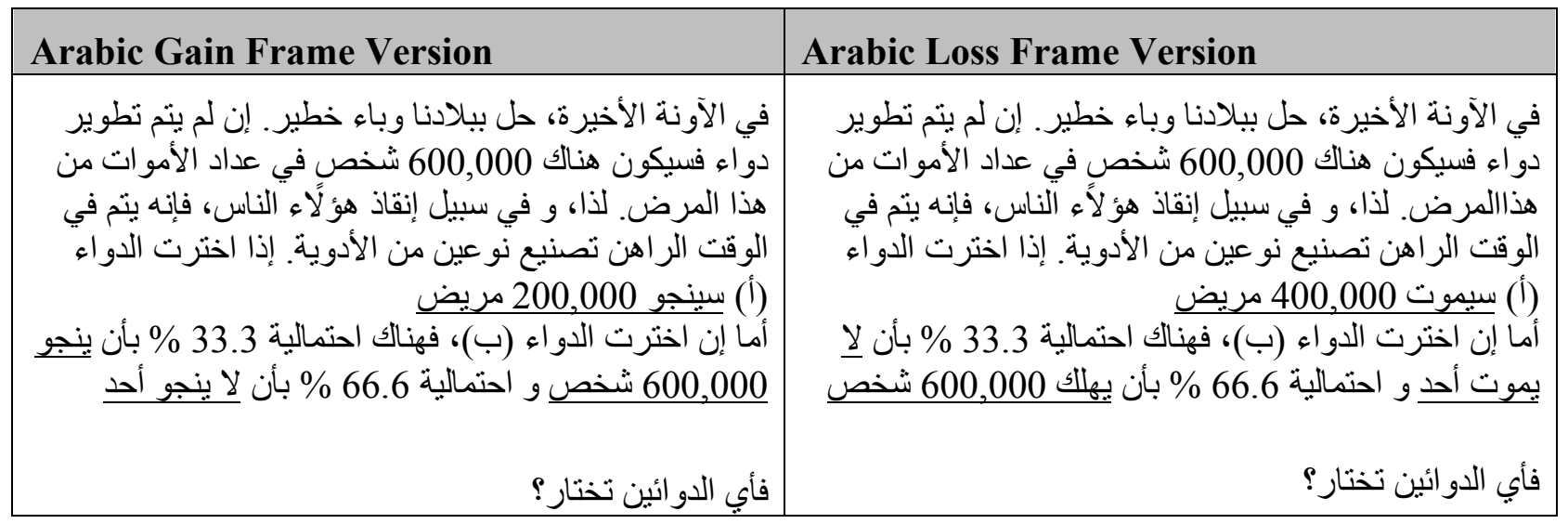

Table 2. Asian Disease problem in Arabic (Translation of Table 1) 
2.1. EXPERIMENT 1. We recruited students in the German Jordanian University $(\mathrm{N}=117,45$ female, average age $=20.33$ ). Their average AoA for English was 4.33. The average selfreported proficiency rating for Arabic was 7.6 and for English was 8.63. The mean response time was 168.25 seconds for Arabic surveys and 193.38 seconds for English surveys.

2.2. EXPERIMENT 2. We recruited students in Philadelphia University $(N=137,27$ female, average age $=21.27$ ). Their average AoA for English was 7.67. The average self-reported proficiency rating for Arabic was 8.5 and for English was 6.13. The mean response time was 110.33 seconds for Arabic surveys and 117.09 seconds for English surveys.

3. Results. Experiment 1 showed a presence of the framing effect regardless of language. This was evident in the asymmetry of choices in Arabic $(\chi 2(1, \mathrm{~N}=60)=3.30, \mathrm{p}>0.05(0.07))$ and English $\left(\chi^{2}(1, \mathrm{~N}=58)=4.14, \mathrm{p}<0.05\right)$. The Arabic case is marginally significant since its $\mathrm{p}$ value is less than 0.1 . See figure 1 .

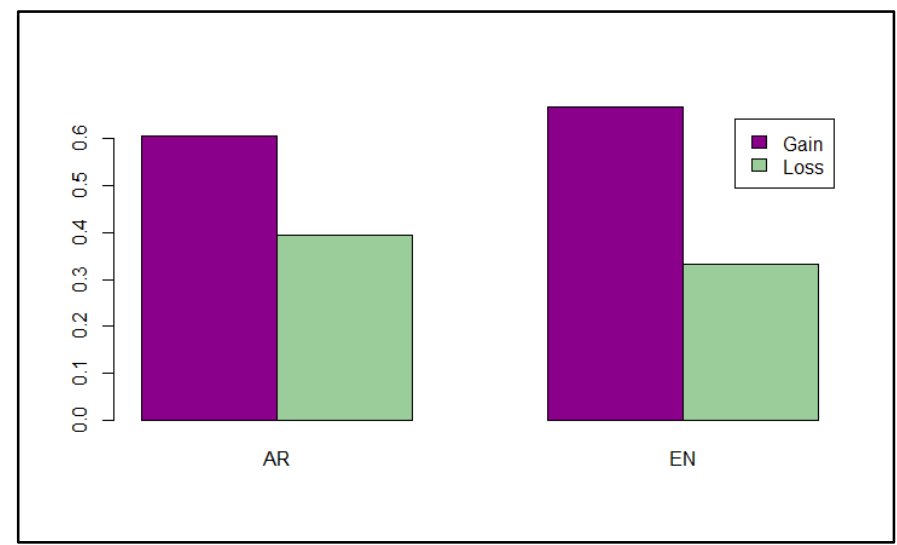

Figure 1. The percentage of participants choosing medicine $\mathrm{A}$ in the First Experiment $(\mathrm{AoA}=$ 4.33), where AR stands for Arabic and EN stands for English.

As for Experiment 2, the asymmetry in choice was observed only in the L1 Arabic language ( $\chi 2$ $(1, \mathrm{~N}=69)=9.03, \mathrm{p}<0.05)$ but was absent in L2 English $(\chi 2(1, \mathrm{~N}=68)=0.07, \mathrm{p}>0.05)$. See figure 2 . This is the same result observed by KHA.

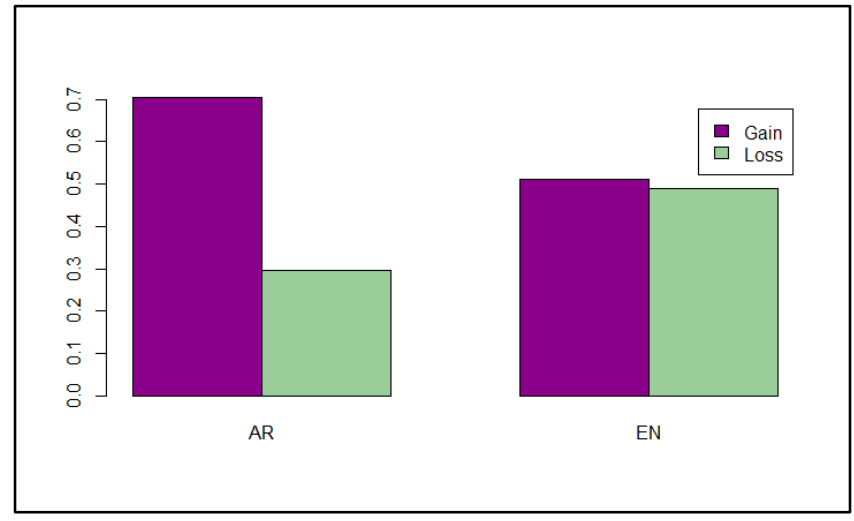

Figure 2. The percentage of participants choosing medicine $\mathrm{A}$ in the Second Experiment. $(\mathrm{AoA}=$ 6.67), where AR stands for Arabic and EN stands for English.

The framing effect was not evident with AoA as early as 4.33 , but was observed with an average AoA of 7.67. The average AoA value of the first experiment was significantly lower than 
that for the second experiment $(\mathrm{t}=8.64, \mathrm{p}<0.05)$. The value obtained in experiment 2 still fell before middle childhood as in experiment 1 . Nevertheless, the decision bias vanished in L2 as was the case in KHA's experiments where the AoA was around adolescence and beyond. As for the proficiency factor, the mean self-reported proficiency value of 8.6 in L2 English out of 10 in Experiment 1 was significantly higher than mean value 6.3 in Experiment $2(t=11.27, p<0.05)$.

4. Discussion. A body of research seems to converge on the detection of emotionality difference in bilinguals between their native and foreign language (Caldwell-Harris, 2015). An earlier intuition about this phenomenon was expressed by German writer Friedrich Sander (quoted in Weinreich, 1953): "Every language establishes an articulated system, a very definite, relatively uniform and closed orientation of perception, feeling, and thinking in those who speak it. The consequence is that the inner attitudes which are conditioned by language will not stand unconnectedly beside one another." Sanders also suggested that there is a bilingualism of feeling.

KHA conducted their Asian Disease experiment with the intuition that, unlike the prediction of Prospect Theory, people will transcend the framing effect and show equivalent likelihood of choice in their L2 for one or the other medicine options in the modified Asian Disease dilemma (Table 1). That's what was confirmed in their three bilingual samples whose average AoA was around and beyond puberty. In our experiments on the Asian Disease problem, the framing effect persisted in L2, with our sample L2 AoA being in early childhood (around 4) and with a high self-reported proficiency (8.63). So we sought a new sample with a potentially lower proficiency (6.13) and a higher L2 AoA (7.67). But to see which factor of these two can be associated with reduced autonomous arousal we found that AoA is more reliably measured in this research. Selfreported proficiency is not very conclusive about actual proficiency (Pavlenko, 2014). In addition, it was found that self-reported proficiency in L2 is dependent on AoA where the earlier a bilingual acquired a language, the higher they would tend to rate their own proficiency in that language (Dewaele, 2009).

Another factor we considered was the amount of time that the participants spent on the Qualtrics survey where a longer time may indicate more deliberation and less time more autonomous responses. However, we did not find a significant difference in the time duration spent on the survey between the two languages in Experiment $2(t=0.64, p>0.05)$. This reduced the possibility that the elicited rationality as indicated by absence of decision bias in L2 was a result of more time taken for deliberation about the dilemma. It was suggested that bilinguals experience purely semantic processing in L2 but affective interference in L1 (Pavlenko, 2012). But we have not referred to research that measured semantic versus affective elements in processing.

One factor that could be considered in future research is the Length of Exposure (LoE) to L2. LoE is not as easy to assess with a retrospective online survey. For participants who learned their L2 in the classroom, we cannot make the confident assumption that if they had comparable AoA then LoE will follow in comparability. It could depend on factors like how much of a priority their schools gave to the L2, what proportion of the school schedule it occupied, and did they use L2 in learning other subjects like science or social sciences.

5. Conclusions. We propose that the presence of the framing effect in L1 is due to higher arousal when viewing a moral dilemma involving death and survival. In a foreign language, the dilemma is a finite problem without additional connotations that may involve affect in the process of decision making. In addition, L2 speakers may approach the level of autonomous arousal in reaction to content in that language in a way comparable to its native speakers with an early enough AoA (including 4 as shown by our sample). Puberty is too high of a threshold for loss of that similari- 
ty in affective processing between L1 and L2, however. The threshold for that can be as early as

8 as indicated by our second sample.

\section{References}

Baron, Jonathan, 1993. Heuristics and biases in equity judgements: A utilitarian approach. In Barbara A. Mellers, \& Jonathan Baron (eds.), Psychological perspectives on justice. Theory and applications. 109-137. New York: Cambridge University Press.

Caldwell-Harris, Catherine L. 2015. Emotionality differences between a native and foreign language: Implications for everyday life. Current Directions in Psychological Science 24(3). 214-219. https://doi.org/10.1177/0963721414566268.

Costa, Albert, Alice Foucart, Sayuri Hayakawa, Melina Aparici, Jose Apesteguia, Joy Heafner \& Boaz Keysar. 2014. Your morals depend on language. PLoS ONE 9(4). e94842. https://doi.org/10.1371/journal.pone.0094842.

Dewaele, Jean-Marc. 2009. Age effects on self-perceived communicative competence and language choice among adult multilinguals. Eurosla Yearbook 9(1). 245-268. https://doi.org/10.1075/eurosla.9.12dew.

Greene, Joshua \& Jonathan Haidt. 2002. How (and where) does moral judgement work? Trends in Cognitive Sciences 6(12). 517- 523.

Haidt, Jonathan. 2007. The new synthesis in moral psychology. Science 316(5827). 998-1002. https://doi.org/10.1126/science.1137651.

Kahneman, Daniel \& Amos Tversky. 1979. Prospect theory: An analysis of decision under risk. Econometrica 47(2). 263- 291. https://doi.org/10.2307/1914185.

Keysar, Boaz, Sayuri L. Hayakawa \& Sun Gyu An. 2012. The foreign-language effect: Thinking in a foreign tongue reduces decision biases. Psychological Science 23(6). 661-668. https://doi.org/10.1177/0956797611432178.

Pavlenko, A. 2012. Affective processing in bilingual speakers: Disembodied cognition? International Journal of Psychology 47(6). 405-428. http://dx.doi.org/10.1080/00207594.2012.743665.

Pavlenko, Aneta. 2014. The bilingual mind: And what it tells us about language and thought. Cambridge: Cambridge University Press.

Tversky, Amos \& Daniel Kahneman. 1981. The framing of decision and the psychology of choice. Science, New Series 212(4481). 453-458. http://www.jstor.org/stable/1685855. 